\title{
Influence of Radiographic Parameters on Reduction of the Critical Shoulder Angle With Arthroscopic Lateral Acromioplasty-A Mathematical Model
}

\author{
J. Christoph Katthagen, M.D., Philip-C. Nolte, M.D., M.A., Gilbert Moatshe, M.D., \\ Grant J. Dornan, M.S., and Peter J. Millett, M.D., M.Sc.
}

\begin{abstract}
Objectives: To develop a mathematical model for the preoperative planning of arthroscopic lateral acromioplasty (ALA) and to evaluate the role of radiographic parameters with regards to the critical shoulder angle (CSA). Methods: Anteroposterior (AP) radiographs of patients who underwent rotator cuff surgery were screened to identify true AP radiographs. Radiographs were assessed for (1) native CSA, (2) CSA after simulated resection of a spur if present, (3) amount of ALA necessary to achieve a CSA of $34^{\circ}$, (4) CSA after 5-mm ALA, (5) lateral acromion angle, (6) acromion index, and (7) sclerosis of the greater tuberosity. Results: A total of 1191 radiographs were screened. Of the 124 patients included, the native CSA was large ( $\left.\geq 35^{\circ}\right)$ in 56 patients $(45 \%)$. In 30 patients $(24 \%)$, a subacromial spur was detected and resection reduced the CSA by a median of $2^{\circ}$. Spur resection alone reduced the CSA to $\leq 34^{\circ}$ in 19 patients $(15.3 \%)$. Mean amount of ALA to achieve a CSA of $34^{\circ}$ was $3.9 \pm$ $1.8 \mathrm{~mm}$, and this value strongly correlated with the CSA before ALA $(\mathrm{R}=0.88, P<.001)$. The linear regression model to determine the amount of ALA to achieve a CSA of $34^{\circ}$ was as follows: Required ALA in $m m=-39.120+1.165 *$ CSA $A_{\text {native }}$ The multiple $\mathrm{R}^{2}$ for this model was 0.777 . Mean reduction of CSA by 5 -mm ALA was $3.8 \pm 0.8^{\circ}$ and $75 \%$ of large CSAs were reduced to a CSA of $30-34^{\circ}$. The acromion index had no significant independent influence on the model $(P=.427)$, whereas lateral acromion angle was an independently significant predictor of required ALA to achieve a CSA of 34 $(P=.019)$. Sclerosis of the greater tuberosity was significantly associated with a CSA of $35^{\circ}$ or greater $(P=.003)$. Conclusions: The amount of ALA needed to reduce a large CSA to $34^{\circ}$ correlates with the CSA before ALA and can preoperatively be planned with the use of a simple equation. Level of Evidence: Level III; cross-sectional design; epidemiology study.
\end{abstract}

B ony scapular morphology has shown association with degenerative rotator cuff tears (RCTs), and various study groups have investigated different measuring techniques. ${ }^{1-3}$ A large lateral extension of the acromion as measured by the acromion index $(\mathrm{AI})$, an increased slope of the acromion in the frontal plane as measured by the lateral acromion angle (LAA), and an upwardly tilted glenoid were found to play an important role in full-thickness RCTs (Fig 1). 1,3,4

From the Department of Trauma, Hand and Reconstructive Surgery, University Hospital Münster, Münster, Germany (J.C.K.); Steadman Philippon Research Institute, Vail, Colorado, U.S.A. (J.C.K., P.-C.N., G.M., G.J.D., P.J.M.); Department of Trauma and Orthopedic Surgery, BG Trauma Center Ludwigshafen, Ludwigshafen, Germany (P.-C.N.); Oslo University Hospital and OSTRC, Norwegian School of Sports Sciences, Oslo, Norway (G.M.); and The Steadman Clinic, Vail, Colorado, U.S.A. (P.J.M.).

The authors report the following potential conflicts of interest or sources of funding: J.C.K. reports personal fees from Depuy Synthes, Germany and personal fees/ research grant from Arthrex, Germany P.-C.N. reports personal fees from Arthrex, Germany, outside the submitted work. P.J.M. reports grants, personal fees, and other from Arthrex; other from Smith $\theta$ Nephew; other from Siemens; other from Ossur; personal fees from Medibridge; personal fees from Springer Publishing; and other from VuMedi, outside the
The critical shoulder angle (CSA) combines the AI and glenoid inclination and is measured by a line connecting the inferior and superior bony margins of the glenoid and a line connecting the inferior bony margin of the glenoid with the most inferolateral part of the acromion (Fig 1). ${ }^{2}$ The CSA is best measured on true anteroposterior (AP) radiographs ${ }^{5}$; however, magnetic resonance imaging and computed tomography also are reliable assessment tools. ${ }^{6,7}$

submitted work. Full ICMJE author disclosure forms are available for this article online, as supplementary material.

J. Christoph Katthagen, M.D., and Philip C. Nolte, M.D., are first authors.

Research was performed at the Steadman Philippon Research Institute, Vail, Colorado. U.S.A.

Received August 31, 2020; accepted January 25, 2021.

Address correspondence to Peter J. Millett, M.D., M.Sc., Steadman Philippon Research Institute, The Steadman Clinic, 181 W Meadow Dr., Ste 400, Vail, CO 81657.E-mail:drmillett@thesteadmanclinic.com

(C) 2022 THE AUTHORS. Published by Elsevier Inc. on behalf of the Arthroscopy Association of North America. This is an open access article under the CC BY-NC-ND license (http://creativecommons.org/licenses/by-nc-nd/4.0/).

2666-061X/201481

https://doi.org/10.1016/j.asmr.2021.01.021 
Whereas a normal CSA is considered to range from $30^{\circ}$ to $34^{\circ}$, an angle of $\geq 35^{\circ}$ has shown association with RCTs $^{7-11}$ and an angle below $30^{\circ}$ has shown association with osteoarthritis. ${ }^{2,7,10,11}$ Moor et al. ${ }^{2}$ postulated that a CSA $>35^{\circ}$ may be associated with RCTs, as a long acromion would lead to an antagonistic direction of pull of the deltoid muscle compared with the supraspinatus muscle. In addition, Gerber et al. ${ }^{12}$ found that a larger CSA would increase the ratio of joint shear-tocompression force, therefore necessitating an increased supraspinatus load. Furthermore, a large CSA may be associated with a greater retear rate following rotator cuff repair. ${ }^{13,14}$ Thus, reduction of a large CSA to a "favorable" range of $30^{\circ}$ to $34^{\circ}$ may potentially prevent progression of rotator cuff tears or protect rotator cuff repair from re-tear. ${ }^{15}$ Furthermore, subacromial spurs and sclerosis of the greater tuberosity are commonly encountered in shoulders with rotator cuff tears. ${ }^{16-18}$ A subacromial osteophyte may enlarge the CSA and should therefore be included in measurement and therapy.

Recently, arthroscopic lateral acromioplasty (ALA) was found to effectively reduce the CSA without violating the acromial deltoid origin following resection of up to $10 \mathrm{~mm} \cdot{ }^{19,20}$ More specifically, an ALA of $5 \mathrm{~mm}$ has been demonstrated to reduce the CSA to $35^{\circ}$ or less in more than $90 \%$ of cases. ${ }^{21}$ However, in patients with a very large CSA $\left(\geq 38^{\circ}\right)$, the recommended amount of $5 \mathrm{~mm}$ ALA may not reduce the CSA to a favorable range. ${ }^{21,22}$ Conversely, in patients with a CSA on the lower end of this range, ALA of $5 \mathrm{~mm}$ could lead to overcorrection of the CSA. Thus, it would be beneficial to have a tool that helps in preoperative planning to individually calculate the amount of resection needed to achieve a CSA that is in the favorable range. The purposes of this study were to develop a mathematical model for preoperative planning of ALA and to evaluate the role of radiographic parameters with regards to the CSA.

\section{Methods}

This research was approved by a local institutional review board (Vail Health Hospital IRB, Protocol \#2020-17). A total of 1191 preoperative shoulder AP radiographs of patients who underwent primary arthroscopic rotator cuff surgery at our institution (P.J.M.) between December 2007 to March 2016 were screened. Calibrated true AP radiographs ${ }^{7,14}$ with fully visible glenohumeral and subacromial joint spaces, and the coracoid tip projected on superomedial quadrant of the humeral head were included (type A according to the Suter-Henninger classification system) (Fig 2). ${ }^{5}$ Duplicated studies, patients with a history of previous shoulder surgery, and patients with previous fracture of the proximal humerus or scapula were excluded.

\section{Radiographic Assessment}

The medical imaging program Merge PACS (IBM Corp., Armonk, NY) was used for radiographic measurements. One fellowship-trained orthopaedic surgeon (J.C.K.) evaluated the preoperative radiographs in the coronal plane. Measurements were only taken once, as intra- and interrater reliability for the CSA have been shown to be good to excellent. ${ }^{2,8,11}$ Radiographs were assessed for (1) the native CSA (Fig 1), ${ }^{2}$ (2) the CSA after theoretical resection of a spur if present (Fig 3), (3) the amount of ALA necessary to achieve a CSA of $34^{\circ}$, which is the upper limit of the favorable zone (Fig 4), ${ }^{15}$ (4) the CSA after simulated $5 \mathrm{~mm}$ ALA representing the clinically recommended amount (Fig 4 ), ${ }^{21}$ (5) the LAA (Fig 1) ${ }^{1}$, (6) the AI (Fig 1), ${ }^{3}$ and (7) the existence of sclerosis of the greater tuberosity and/or subacromial spurs (Figs 2 and 3). According to Oh et al., ${ }^{23}$ heel-type, traction-type, and bird beaktypes were included.

\section{Statistical Analysis}

Simple and multiple linear regression were performed to predict the ALA amount required to achieve a postoperative CSA less than $35^{\circ}$ as a function of the preoperative (native) CSA. The multipredictor model was built to adjust for LAA and AI while estimating the independent effect of preoperative CSA on the required ALA resection. The explanatory power of the regression models was assessed using the R-squared statistic. Both a confidence interval for the regression fit and a prediction interval for individual shoulders were presented graphically. Residual diagnostics were assessed to ensure model fit and that model assumptions were satisfied. Association between binary variables was assessed using the Fisher exact test, whereas association between continuous covariates was assessed using Pearson's correlation. $P$ values $<.05$ were interpreted as statistically significant. All statistical analysis and plots were produced using the statistical programming language $\mathrm{R}$, version $4.0 .0 .^{24}$

\section{Results}

High-quality calibrated true AP radiographs of 124 patients ( 124 shoulders; 86 men and 38 women) with a mean age of 57.7 years (range, 25-78 years) were included in the radiographic analysis. The mean CSA was $33.9^{\circ}\left(\right.$ range, $25^{\circ}-43^{\circ}$ ) and was $35^{\circ}$ or greater in 56 patients $(45 \%)$. In $24 \%$ of patients $(\mathrm{n}=30)$ a subacromial spur was detected (Fig 3). Sclerosis of the greater tuberosity was observed in 56 patients $(45 \%)$ (Fig 2). Summaries of demographic and radiographic characteristics are presented in Table 1.

The simulated resection of the subacromial spur reduced the CSA by a median of $2^{\circ}$ (range, 1-4 $4^{\circ}$ among 30 patients $(24.2 \%)$ where a subacromial spur was found (Fig 3). Spur resection alone was sufficient to 


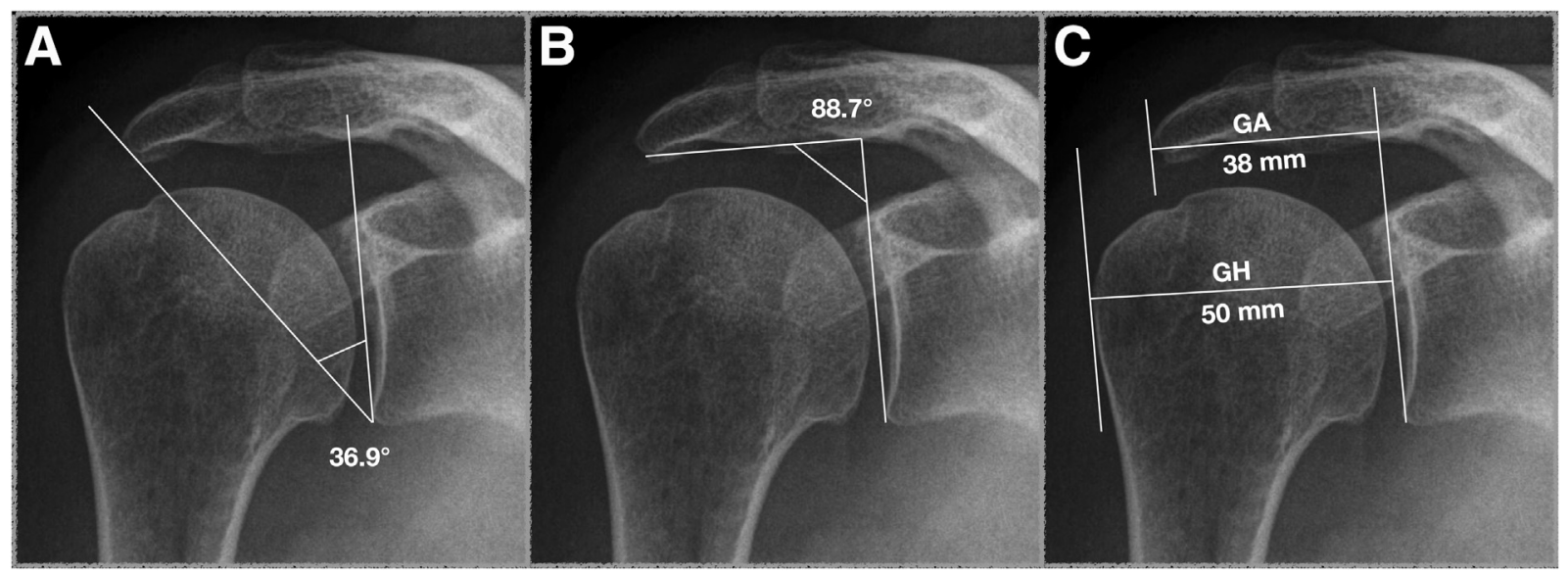

Fig 1. Illustration of measurement techniques for the CSA, LAA, and AI. (A) The CSA is measured by a line connecting the inferior and superior bony margins of the glenoid and a line connecting the inferior bony margin of the glenoid with the most inferolateral part of the acromion. In this case, the CSA is $36.9^{\circ}$. (B) The LAA is formed by the intersection of a line parallel to the undersurface of the acromion and a line connecting the inferior and superior bony margins of the glenoid. In this case the LAA is $88.7^{\circ}$. (C) The AI is measured by the distance between glenoid and lateral acromion (GA) divided by distance between glenoid and lateral humerus $(\mathrm{GH})$. In this case the $\mathrm{AI}$ is $0.76(\mathrm{AI}=38 \mathrm{~mm} / 50 \mathrm{~mm}=0.76)$. (AI, acromion index; CSA, critical shoulder angle; LAA, lateral acromion angle.)

reduce the CSA to $\leq 34^{\circ}$ in 19 patients $(15.3 \%)$. The mean amount of ALA needed to achieve a CSA of $34^{\circ}$ was $3.9 \pm 1.8 \mathrm{~mm}$, and this value strongly correlated with the CSA before ALA $(\mathrm{R}=0.88, P<.001$; Fig 5, Table 2). The linear regression model to determine required amount of ALA to achieve a CSA of $34^{\circ}$ was

$$
\text { Required ALA in } \mathrm{mm}=-39.120+1.165 * C S A_{\text {native }}
$$

The multiple $\mathrm{R}^{2}$ for this model was 0.777 , indicating that nearly $78 \%$ of the variation in required lateral acromioplasty amount is explained by preoperative CSA. The mean reduction of the CSA by a simulated $5-\mathrm{mm}$ ALA was $3.8 \pm 0.8^{\circ}$ and $75 \%$ of large CSAs were reduced to the favorable CSA range by $5 \mathrm{~mm}$ ALA (Fig 4). When we adjusted for native CSA, the LAA (mean 80'; range $67-94^{\circ}$ ) was also an independently significant predictor of required ALA to achieve a CSA of $34^{\circ}(P=.019)$ (Fig 1). However, this expanded model achieved only a marginally improved R-squared value of 0.805 compared with the single covariate regression model and thus did not add substantially to the clinical relevance of the model. Meanwhile, AI had no significant independent influence on the model $(P=.427)$ (Fig 1).
Fig 2. Inclusion and exclusion criteria for AP radiographs. (A) Included true AP radiograph. (1) Fully visible subacromial and (2) glenohumeral joint spaces, and the (3) coracoid tip projected on superomedial quadrant of the humeral head. Dotted area represents the anterior and posterior glenoid rims which are overlapping. (B) Excluded radiograph. (1) Subacromial and (2) glenohumeral joint spaces not fully visible, and the (3) coracoid tip not projected on superomedial quadrant of the humeral head. Dotted area represents the anterior and posterior glenoid rims which are not overlapping suggesting that this radiograph is not true AP. Asterisk indicates sclerosis of greater tuberosity. (AP, anteroposterior.)

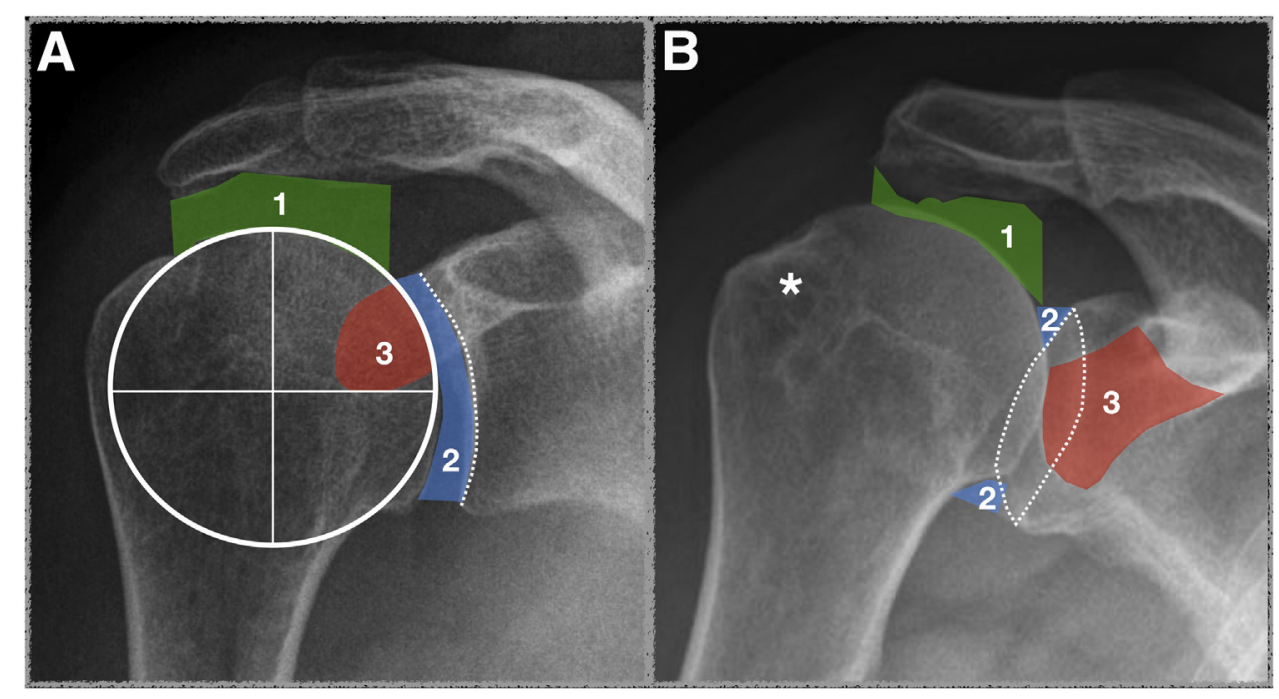




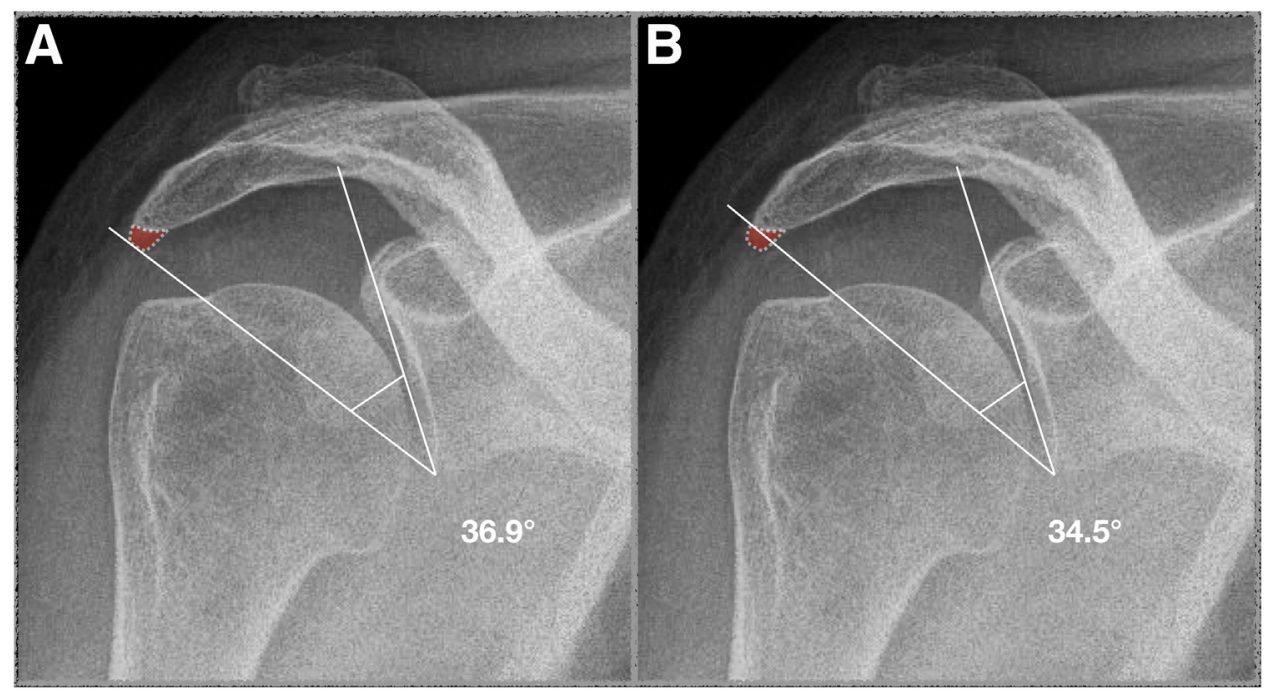

Fig 3. Effect of spur resection on CSA. (A) The native CSA with a traction-type spur (dotted area) is $36.9^{\circ}$. (B) Simulated resection of the spur (dotted area) results in a CSA of $34.5^{\circ}$. (CSA, critical shoulder angle.)

Sclerosis of the greater tuberosity was significantly associated with a CSA of $35^{\circ}$ or larger (odds ratio $3.22,95 \%$ confidence interval 1.41-7.55, $P=.003$ ) (Fig 2).

\section{Discussion}

The most important finding of this study was that the amount of ALA needed to reduce a large CSA to $34^{\circ}$ $(3.9 \pm 1.8 \mathrm{~mm})$ strongly correlates with the CSA before ALA and can preoperatively be planned on a true AP radiograph by using a simple equation with minimal uncertainty. This finding could potentially aid surgeons in preoperative planning of ALA. In addition, the presence of a subacromial spur increased the CSA and sclerosis of the greater tuberosity was significantly associated with a CSA of $\geq 35^{\circ}$.

Bony morphology of the scapula has long been suspected to play an important role in the pathogenesis of
RCTs. ${ }^{16,25}$ Numerous measuring techniques have been introduced, with the goal to show association with rotator cuff pathology. ${ }^{1-4}$ The CSA described by Moor et al. ${ }^{2}$ has received a great deal of scientific attention since it was introduced in 2013. By combining the lateral extension of the acromion ${ }^{3}$ and glenoid inclination, ${ }^{4}$ both of which individually have been shown to be associated with RCTs, the CSA is potentially superior to previous measurements. ${ }^{7,11}$

A large CSA is considered to be $35^{\circ}$ or greater and has shown association with rotator cuff tears, ${ }^{7-11}$ whereas a small CSA lower than $30^{\circ}$ has shown association with osteoarthritis. $^{2,7,11}$ When performing rotator cuff repair, an ALA may be considered to reduce the CSA to protect the repair from retear. Gerber et al. ${ }^{14}$ have demonstrated that ALA is a safe adjunct to rotator cuff repair and that the CSA should be reduced to $33^{\circ}$ or less to avoid retears in patients with large CSAs. This is in
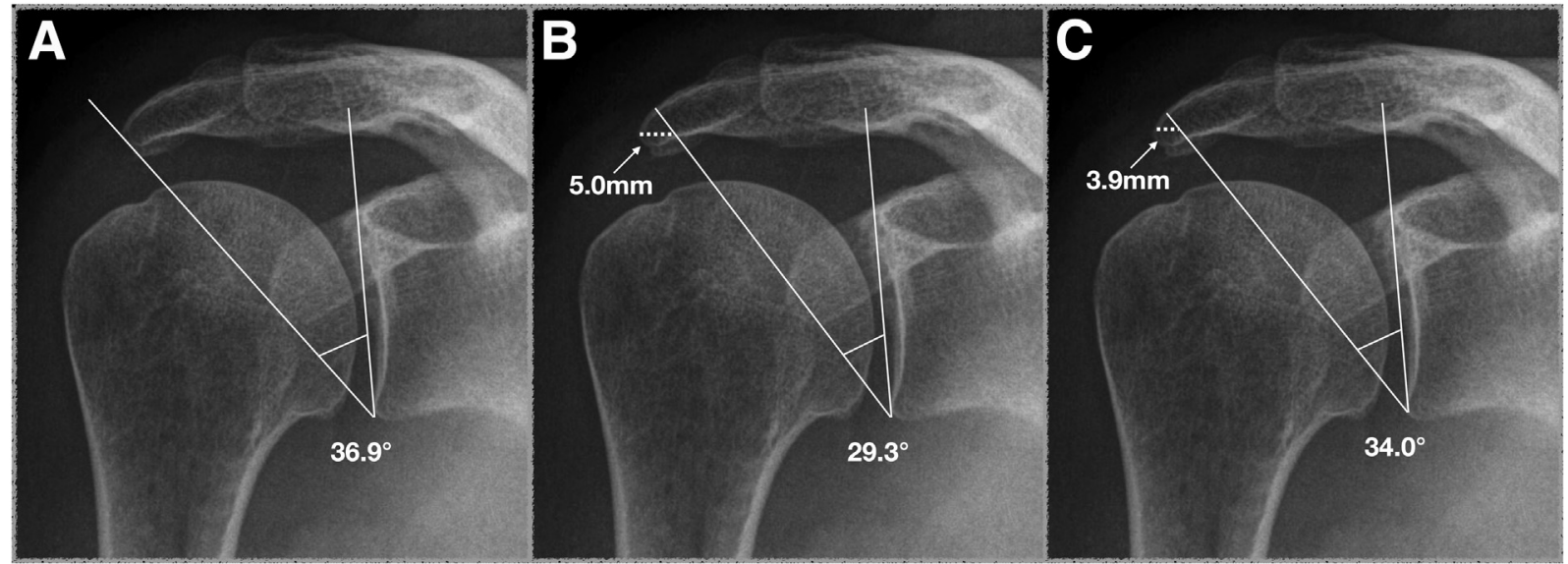

Fig 4. Simulated lateral acromioplasty. (A) Native CSA. (B) The CSA is reduced to $29.3^{\circ}$ after 5 mm (dotted line) of simulated lateral acromioplasty. (C) Simulated lateral acromioplasty of $3.9 \mathrm{~mm}$ (dotted line) was necessary to reduce the CSA from $36.9^{\circ}$ to $34.0^{\circ}$. (CSA, critical shoulder angle.) 
Table 1. Patient Characteristics

\begin{tabular}{lc}
\hline \multicolumn{1}{c}{ Variable } \\
\hline $\mathrm{N}$ & 124 \\
Male, n (\%) & $86(69.4)$ \\
Age, y, mean (range) & $57.7(25-78)$ \\
$\mathrm{CSA},{ }^{\circ}$ mean (range) & $33.9(25-43)$ \\
$\mathrm{CSA} \geq 35^{\circ}, \mathrm{n}(\%)$ & $56(45)$ \\
Native CSA among patients with & $37(35-43)$ \\
CSA $\geq 35^{\circ}$, median (range) & \\
Greater tuberosity sclerosis, n (\%) & $56(45)$ \\
Subacromial spur, n (\%) & $30(24)$ \\
\hline
\end{tabular}

CSA, critical shoulder angle.

line with a recent systematic review that has demonstrated that there may be an association of a greater CSA with retears. ${ }^{13}$ However, indications for ALA do not only involve rotator cuff repair: Katthagen and Millett ${ }^{15}$ suggested that ALA should be considered for patients with a CSA $>38^{\circ}$ if the rotator cuff is at risk (severe tendinopathy or partial-thickness tears $>50 \%$ ) when operatively treating subacromial impingement syndrome.

Although performing ALA to reduce the CSA to a favorable range is often supported, there remains a paucity on how much of the lateral acromion to resect. One of the main concerns is that the deltoid insertion might be injured, thus leading to weakness and/or rupture. Gerber et al. ${ }^{14}$ resected 3 to $8 \mathrm{~mm}$ of the lateral acromion in 49 shoulders to achieve a favorable CSA according to the preoperative measurement. No significant damage to the deltoid insertion was found in their investigation. Katthagen et al. ${ }^{19}$ showed, that a lateral acromion resection of $5 \mathrm{~mm}$ significantly reduces the CSA and did not damage the deltoid origin. These findings were supported by Marchetti et al. ${ }^{20}$ who demonstrated that ALA of up to $10 \mathrm{~mm}$ neither reduced the deltoid's failure load nor did any macroscopic changes occur. Our findings that a mean ALA of $3.9 \pm$ $1.8 \mathrm{~mm}$ is needed to achieve a favorable CSA of $34^{\circ} \mathrm{can}$ therefore be considered safe in the light of previous research. According to our equation, only a CSA of $\geq 43^{\circ}$ would lead to a potentially critical ALA over 10 $\mathrm{mm}$. In this case, we believe, that it should be carefully evaluated whether an ALA of more than $10 \mathrm{~mm}$ is necessary and outweighs the risks of injury to the deltoid insertion. However, a CSA of this magnitude is rather uncommon.

Our second finding was, that subacromial spurs increased the CSA by a median of $2^{\circ}$ and the resection of theses spurs alone was sufficient to reduce the CSA to the favorable range of 30 to $35^{\circ}$ in some cases. Subacromial spurs have been previously shown to be associated with RCTs. ${ }^{17,18}$ Ogawa et al. ${ }^{17}$ observed $69 \%$ of large spurs in cases with bursal-sided tears, complete tears of the supraspinatus and massive rotator cuff tears. Similarly, Oh et al. ${ }^{23}$ found a relationship between subacromial osteophytes and full-thickness rotator cuff tears, such that subacromial spurs were found in $78 \%$ of patients with full-thickness tears, as opposed to only $58 \%$ in the control group. A potential reason for the quantitative differences compared to our study is that Oh et al. ${ }^{23}$ also used images in the sagittal plane (e.g., sagittal magnetic resonance imaging/supraspinatus outlet view) therefore identifying spurs that we were not able to detect in coronal plane (AP) radiographs. One of the early studies to investigate subacromial spurs in relationship to the CSA proposed that RCTs were affected more by osteophytes than by the CSA when evaluated together as risk factor. ${ }^{18}$ In cases with a high CSA of more than $38^{\circ}$, patients without an osteophyte had RCTs in $76.9 \%$ and patients with an osteophyte had RCTs in $92.3 \%$ of cases. ${ }^{18}$ However, this study did not explicitly state if the CSA was measured with or without including the subacromial spur. In the present study a subacromial spur, if present, was included in the CSA measurement and its resection may be sufficient to correct the CSA to favorable values. However, if the spur is not included in the CSA measurement and ALA is planned according to the CSA, this could potentially lead to under-resection intraoperatively.

Interestingly, we found that sclerosis of the greater tuberosity was identified as indicator for a large CSA. In

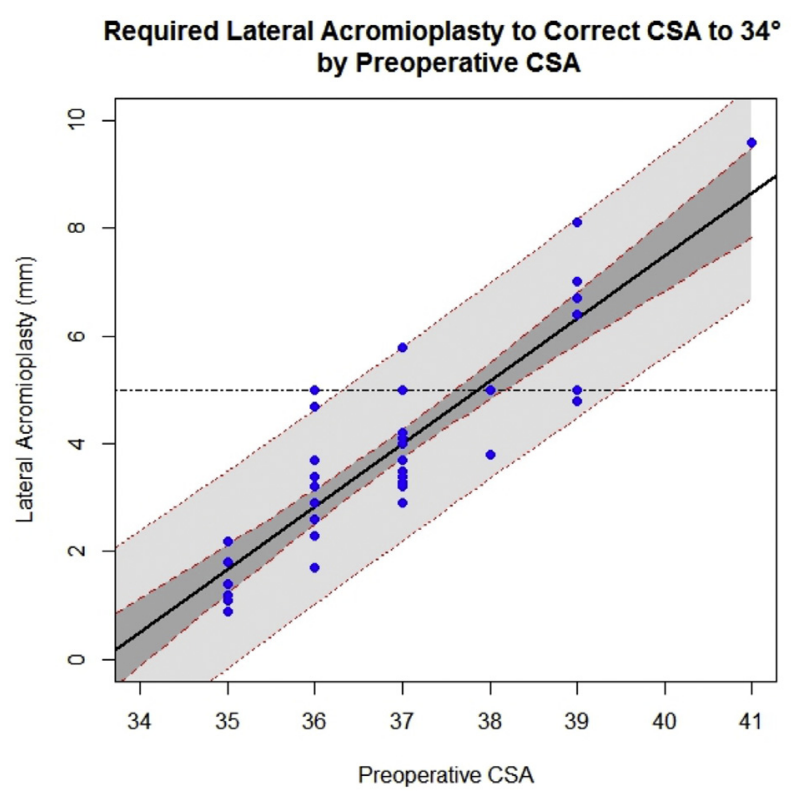

Fig 5. Relationship between the CSA and amount of arthroscopic lateral acromioplasty required to achieve a CSA of $34^{\circ}$. The solid black line represents linear regression fit while the dark gray region denotes the $95 \%$ confidence interval for the regression line and the light gray region denotes the $95 \%$ prediction interval for individual patients. (CSA, critical shoulder angle.) 
Table 2. Required Resection of the Lateral Acromion According to the Native CSA to Achieve a CSA of $34^{\circ}$

\begin{tabular}{ll}
\hline Native CSA, ${ }^{\circ}$ & Required Resection, $\mathrm{mm}$ \\
\hline 36 & 2.82 \\
37 & 3.985 \\
38 & 5.15 \\
39 & 6.315 \\
40 & 7.48 \\
\hline
\end{tabular}

CSA, critical shoulder angle.

shoulders with a large CSA, sclerosis may be the result of mechanical conflict between acromion and greater tuberosity. If this radiographic parameter is encountered, one should think of a large CSA that may require treatment in the setting of RCT.

\section{Limitations}

We recognize that our study has some weaknesses. First, a limitation of the CSA itself is that it does not consider the morphology of the humeral head and the greater tuberosity. The CSA is also dependent on true AP radiographs, and even slight changes in scapula alignment can lead to a variability in CSA. ${ }^{5}$ For this reason, computed tomography scans may have been more accurate. However, we tried to minimize this potential conflict by only using true AP shoulder radiographs with fully visible glenohumeral and subacromial joint spaces, and the coracoid tip projected on superomedial quadrant of the humeral head. Second, the measurements of the CSA were only taken once by an orthopaedic surgeon, and we did not evaluate for intra- and interrater reliability. However, we believe that this was not necessary, as it has been demonstrated numerous times that the CSA shows good to excellent intra- and interrater reliability. ${ }^{2,8,11}$ Third, there may be a selection bias as we have only used true AP radiographs (124 of 1191 patients) for CSA measurement according to the criteria described above. Therefore, we might have not included some patients with a large CSA who did not have adequate radiographs. Fourth, the ALA was performed virtually only. This is why we cannot state with certainty that the recommended virtual resection would equally translate to the desired postoperative CSA in the clinical setting or to improvements in postoperative patient outcomes.

\section{Conclusions}

The amount of ALA needed to reduce a large CSA to $34^{\circ}$ correlates with the CSA before ALA and can preoperatively be planned with the use of a simple equation.

\section{References}

1. Banas MP, Miller RJ, Totterman S. Relationship between the lateral acromion angle and rotator cuff disease. J Shoulder Elbow Surg 1995;4:454-461.
2. Moor BK, Bouaicha S, Rothenfluh DA, Sukthankar A, Gerber C. Is there an association between the individual anatomy of the scapula and the development of rotator cuff tears or osteoarthritis of the glenohumeral joint? A radiological study of the critical shoulder angle. Bone Joint J 2013;95-B:935-941.

3. Nyffeler RW, Werner CM, Sukthankar A, Schmid MR, Gerber C. Association of a large lateral extension of the acromion with rotator cuff tears. J Bone Joint Surg Am 2006;88:800-805.

4. Tetreault P, Krueger A, Zurakowski D, Gerber C. Glenoid version and rotator cuff tears. J Orthop Res 2004;22: 202-207.

5. Suter T, Gerber Popp A, Zhang Y, Zhang C, Tashjian RZ, Henninger HB. The influence of radiographic viewing perspective and demographics on the critical shoulder angle. J Shoulder Elbow Surg 2015;24:e149-e158.

6. Bouaicha S, Ehrmann C, Slankamenac K, Regan WD, Moor BK. Comparison of the critical shoulder angle in radiographs and computed tomography. Skeletal Radiol 2014;43:1053-1056.

7. Spiegl UJ, Horan MP, Smith SW, Ho CP, Millett PJ. The critical shoulder angle is associated with rotator cuff tears and shoulder osteoarthritis and is better assessed with radiographs over MRI. Knee Surg Sports Traumatol Arthrose 2016;24:2244-2251.

8. Cherchi L, Ciornohac JF, Godet J, Clavert P, Kempf JF. Critical shoulder angle: Measurement reproducibility and correlation with rotator cuff tendon tears. Orthop Traumatol Surg Res 2016;102:559-562.

9. Moor BK, Rothlisberger M, Muller DA, et al. Age, trauma and the critical shoulder angle accurately predict supraspinatus tendon tears. Orthop Traumatol Surg Res 2014;100: 489-494.

10. Smith GCS, Liu V, Lam PH. The critical shoulder angle shows a reciprocal change in magnitude when evaluating symptomatic full-thickness rotator cuff tears versus primary glenohumeral osteoarthritis as compared with control subjects: A systematic review and meta-analysis. Arthroscopy 2020;36:566-575.

11. Heuberer PR, Plachel F, Willinger L, et al. Critical shoulder angle combined with age predict five shoulder pathologies: A retrospective analysis of 1000 cases. BMC Musculoskelet Disord 2017;18:259.

12. Gerber C, Snedeker JG, Baumgartner D, Viehöfer AF. Supraspinatus tendon load during abduction is dependent on the size of the critical shoulder angle: A biomechanical analysis. J Orthop Res 2014;32:952-957.

13. Docter S, Khan M, Ekhtiari S, et al. The relationship between the critical shoulder angle and the incidence of chronic, full-thickness rotator cuff tears and outcomes after rotator cuff repair: A systematic review. Arthroscopy 2019;35:3135-3143.e3134.

14. Gerber C, Catanzaro S, Betz M, Ernstbrunner L. Arthroscopic correction of the critical shoulder angle through lateral acromioplasty: A safe adjunct to rotator cuff repair. Arthroscopy 2018;34:771-780.

15. Katthagen JC, Millett PJ. Editorial commentary: Lateral acromioplasty is clinically safe and has the potential to reduce the risk for rotator cuff re-tears. Arthroscopy 2018;34:781-783. 
16. Neer CS 2nd. Anterior acromioplasty for the chronic impingement syndrome in the shoulder: A preliminary report. J Bone Joint Surg Am 1972;54:41-50.

17. Ogawa K, Yoshida A, Inokuchi W, Naniwa T. Acromial spur: Relationship to aging and morphologic changes in the rotator cuff. J Shoulder Elbow Surg 2005;14:591-598.

18. Kim JH, Min YK, Gwak HC, Kim CW, Lee CR, Lee SJ. Rotator cuff tear incidence association with critical shoulder angle and subacromial osteophytes. J Shoulder Elbow Surg 2019;28:470-475.

19. Katthagen JC, Marchetti DC, Tahal DS, Turnbull TL, Millett PJ. The effects of arthroscopic lateral acromioplasty on the critical shoulder angle and the anterolateral deltoid origin: An anatomic cadaveric study. Arthroscopy 2016;32: 569-575.

20. Marchetti DC, Katthagen JC, Mikula JD, et al. Impact of arthroscopic lateral acromioplasty on the mechanical and structural integrity of the lateral deltoid origin: A cadaveric study. Arthroscopy 2017;33:511-517.
21. Karns MR, Jacxsens M, Uffmann WJ, Todd DC, Henninger HB, Burks RT. The critical acromial point: The anatomic location of the lateral acromion in the critical shoulder angle. J Shoulder Elbow Surg 2018;27: $151-159$.

22. Hohmann E, Shea K, Scheiderer B, Millett P, Imhoff A. Indications for arthroscopic subacromial decompression. A Level V evidence clinical guideline. Arthroscopy 2020;36: 913-922.

23. Oh JH, Kim JY, Lee HK, Choi JA. Classification and clinical significance of acromial spur in rotator cuff tear: Heel-type spur and rotator cuff tear. Clin Orthop Relat Res 2010;468:1542-1550.

24. R Core Team. R. A language and environment for statistical computing. Vienna, Austria: R Foundation for Statistical Computing, 2019.

25. Bigliani LU, Ticker JB, Flatow EL, Soslowsky LJ, Mow VC. The relationship of acromial architecture to rotator cuff disease. Clin Sports Med 1991;10:823-838. 